\title{
Odd Harmonious Labeling of Some Classes of Graphs
}

\author{
P. JeYANThi ${ }^{1}$ AND S. PhilO ${ }^{2}$ \\ ${ }^{1}$ Research Centre, Department of Mathematics, \\ Govindammal Aditanar College for Women, \\ Tiruchendur - 628 215, Tamil Nadu, India. \\ ${ }^{2}$ Department of Mathematics, \\ St.Xavier's College, Palayamkottai, \\ Tirunelveli-627002, Tamilnadu, India. \\ jeyajeyanthi@rediffmail.com, lavernejudia@gmail.com
}

\begin{abstract}
A graph $G(p, q)$ is said to be odd harmonious if there exists an injection $f: V(G) \rightarrow$ $\{0,1,2, \cdots, 2 q-1\}$ such that the induced function $f^{*}: E(G) \rightarrow\{1,3, \cdots, 2 q-1\}$ defined by $f^{*}(u v)=f(u)+f(v)$ is a bijection. In this paper we prove that $T_{p^{-}}$tree, $T \hat{o} P_{m}$, Tô $2 P_{m}$, regular bamboo tree, $C_{n} \hat{\circ} P_{m}, C_{n} \hat{\circ} 2 P_{m}$ and subdivided grid graphs are odd harmonious.

\section{RESUMEN}

Un grafo $G(p, q)$ se dice impar armonioso si existe una inyección $f: V(G) \rightarrow\{0,1,2, \cdots$, $2 q-1\}$ tal que la función inducida $f^{*}: E(G) \rightarrow\{1,3, \cdots, 2 q-1\}$ definida por $f^{*}(u v)=f(u)+f(v)$ es una biyección. En este artículo probamos que los grafos $T_{p}$-árboles, $T \hat{o} P_{m}, T \hat{o} 2 P_{m}$, árboles bambú regulares, $C_{n} \hat{o} P_{m}, C_{n} \hat{\circ} 2 P_{m}$ y cuadrículas subdivididas son impar armoniosos.
\end{abstract}

Keywords and Phrases: harmonious labeling, odd harmonious labeling, transformed tree, subdivided grid graph, regular bamboo tree.

2020 AMS Mathematics Subject Classification: 05C78. 


\section{Introduction}

Throughout this paper by a graph is implied as a finite, simple and undirected. For standard terminology and notation we follow Harary [3]. A graph $G(V, E)$ with $p$ vertices and $q$ edges is called a $(p, q)$ - graph. The graph labeling is an assignment of integers to the set of vertices or edges or both, subject to certain conditions. An extensive survey of various graph labeling problems is available in [1]. Graham and Sloane [2] introduced harmonious labeling during their study of modular versions of additive bases problems stemming from error correcting codes. A graph $G$ is said to be harmonious if there exists an injection $f: V(G) \rightarrow Z_{q}$ such that the induced function $f^{*}: E(G) \rightarrow Z_{q}$ defined by $f^{*}(u v)=(f(u)+f(v))(\bmod q)$ is a bijection and $f$ is called harmonious labeling of $G$. The concept of an odd harmonious labeling was due to Liang and Bai [14]. A graph $G$ is said to be odd harmonious if there exists an injection $f: V(G) \rightarrow\{0,1,2, \cdots, 2 q-1\}$ such that the induced function $f^{*}: E(G) \rightarrow\{1,3, \cdots, 2 q-1\}$ defined by $f^{*}(u v)=f(u)+f(v)$ is a bijection. If $f: V(G) \rightarrow\{0,1,2, \cdots, q\}$ then $f$ is called as strongly odd harmonious labeling and $G$ is called a strongly odd harmonious graph. The odd harmoniousness of a graph is useful for the solution of undetermined equations. The following results have been proved in [14:

1. If $G$ is an odd harmonious graph, then $G$ is a bipartite graph. Hence any graph that contains an odd cycle is not an odd harmonious.

2. If a $(p, q)$ - graph $G$ is odd harmonious, then $2 \sqrt{q} \leq p \leq(2 q-1)$.

3. If $G$ is an odd harmonious Eulerian graph with $q$ edges, then $q \equiv 0,2(\bmod 4)$.

Followed by this, Vaidya and Shah [18, [19] showed that shadow and splitting graphs are odd harmonious. Selvaraju et al. [17] established that some path related graphs are odd harmonious. Jeyanthi et al. proved that the following graphs are odd harmonious: double quadrilateral snake and banana tree [5], cycle related graphs [6], plus graphs [7], super subdivision graphs [], subdivided shell graphs [9], spider and necklace graphs [10, m-shadow, m-splitting and m-mirror graphs [11] and [12, grid graphs [13].

We use the following definitions in the subsequent section.

Definition 1.1. Let $G=(V, E)$ be a graph. $G$ is called a path $P_{n}$ if $V=\left\{v_{1}, v_{2}, \cdots, v_{n}\right\}$ such that $1 \leq i \leq n,\left(v_{i}, v_{i+1}\right) \in E$.

Definition 1.2. The Cartesian product of graphs $G$ and $H$ denoted as $G \square H$, is the graph with vertex set $V(G) \times V(H)=\{(u, v) \mid u \in V(G)$ and $v \in V(H)\}$ and $(u, v)$ is adjacent to $\left(u^{\prime}, v\right)$ if and only if either $u=u^{\prime}$ and $\left(v, v^{\prime}\right) \in E(H)$ or $v=v^{\prime}$ and $(u, u) \in E(G)$. The Cartesian product of two paths $P_{m}$ and $P_{n}$ denoted by $P_{m} \times P_{n}$ is known as a grid graph on mn vertices and $2 m n-(m+n)$ edges. 
Definition 1.3. Let $G$ be a graph with $p$ vertices and $H$ be any graph and $x$ be a vertex of $H$. A graph $G \hat{o} H$ is obtained from $G$ and $p$ copies of $H$ by identifying vertex $x$ of $i^{\text {th }}$ copy of $H$ with $i^{\text {th }}$ vertex of $G$.

Definition 1.4. 4. Let $T$ be a tree and $u_{0}$ and $v_{0}$ be the two adjacent vertices in $T$. Let $u$ and $v$ be the two pendant vertices of $T$ such that the length of the path $u_{0}-u$ is equal to the length of the path $v_{0}-v$. If the edge $u_{0} v_{0}$ is deleted from $T$ and $u$ and $v$ are joined by an edge uv, then such a transformation of $T$ is called an elementary parallel transformation (or an ept) and the edge $u_{0} v_{0}$ is called transformable edge. If by some sequence of ept's, $T$ can be reduced to a path, then $T$ is called a $T_{p^{-}}$tree (transformed tree) and such sequence regarded as a composition of mappings (ept's) denoted by $P$ is called a parallel transformation of $T$. The path, the image of $T$ under $P$ is denoted as $P(T) . A T_{p^{-}}$tree and the sequence of two ept's reducing it to a path are illustrated in Figure 1.

a) A $T_{p^{-}}$tree $T$

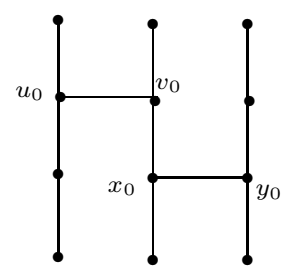

b) An ept $P_{1}(T)$

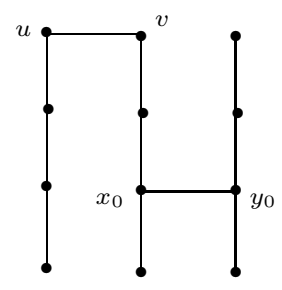

c) Second ept $P_{2}(T)$

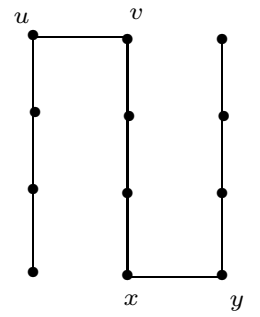

Figure 1: Transformed tree

Definition 1.5. 15] Let $T$ be a $T_{p}$-tree with $n$ vertices $v_{1}, v_{2}, \cdots, v_{n}$. The graph $T \hat{o} P_{m}$ is obtained from $T$ and $n$ copies of $P_{m}$ by identifying a pendant vertex of $i^{\text {th }}$ copy of $P_{m}$ with vertex $v_{i}$ of $T$.

Definition 1.6. 16] Consider $k$ copies of paths $P_{n}$ of length $n-1$ and stars $S_{m}$ with $m$ pendant vertices. Identify one of the two pendant vertices of the $j^{\text {th }}$ path with the centre of the $j^{\text {th }}$ star. Identify the other pendant vertex of each path with a single vertex $u_{0}\left(u_{0}\right.$ is not in any of the star and path). The graph obtained is a regular bamboo tree. 


\section{Main Results}

In this section, we prove that $T_{p^{-}}$tree, $T \hat{o} P_{m}, T \hat{o} 2 P_{m}$, regular bamboo tree, $C_{n} \hat{o} P_{m}, C_{n} \hat{\circ} 2 P_{m}$ and subdivided grid graphs are odd harmonious.

Theorem 2.1. Every $T_{p}$ - tree is strongly odd harmonious.

Proof. Let $T$ be a $T_{p}$-tree with $n$ vertices. By definition, there exists a parallel transformation $P$ of $T$, we have $V(P(T))=V(T)$ and $E(P(T))=\left(E(T)-E_{d}\right) \cup E_{a}$, where $E_{d}$ is the set of deleted edges and $E_{a}$ is the set of newly added edges through the sequence $P=\left(P_{1}, P_{2}, \cdots, P_{l}\right)$ of the ept's used to obtain $P(T)$. Hence $E_{d}$ and $E_{a}$ have the same number of edges. Let $u_{1}, u_{2}, \cdots, u_{n}$ be the vertices of $P(T)$ successively, from one pendant vertex of $P(T)$ right up to the other. This $T_{p}$-tree has $n$ vertices and $n-1$ edges.

We define a labeling $f: V(G) \rightarrow\{0,1,2, \cdots, q=n-1\}$ as follows: $f\left(u_{i}\right)=i-1, \quad 1 \leq i \leq n$.

Let $\left(u_{i} u_{j}\right)$ be an edge of $T, 1 \leq i<j \leq n$. Let the ept $P_{1}$ delete the edge $\left(u_{i} u_{j}\right)$ and adds the edge $\left(u_{i+t} u_{j-t}\right)$ where $t$ is the distance from $u_{i}$ to $u_{i+t}$ and also the distance from $u_{j}$ to $u_{j-t}$. Let the parallel transformation $P$ contain one of the constituent ept's $P_{1}$. Since $\left(u_{i+t} u_{j-t}\right)$ is an edge of $P(T)$, it follows that $i+t+1=j-t$, implies $j=i+2 t+1$.

The induced edge label of $\left(u_{i} u_{j}\right)$ is given by

$f^{*}\left(u_{i} u_{j}\right)=f^{*}\left(u_{i} u_{i+2 t+1}\right)=f\left(u_{i}\right)+f\left(u_{i+2 t+1}\right)=2(i+t)-1$, $f^{*}\left(u_{i+t} u_{j-t}\right)=f^{*}\left(u_{i+t} u_{i+t+1}\right)=f\left(u_{i+t}\right)+f\left(u_{i+t+1}\right)=2(i+t)-1$, $f^{*}\left(u_{i} u_{j}\right)=f^{*}\left(u_{i+t} u_{j-t}\right)$.

The induced edge label is $f^{*}\left(u_{i} u_{i+1}\right)=2 i-1, \quad 1 \leq i \leq n-1$.

Thus the induced edge labels are $1,3, \cdots, 2 n-3$. Therefore every $T_{p}$-tree is strongly odd harmonious.

A strongly odd harmonious labeling of a $T_{p^{-}}$tree with 12 vertices is shown in Figure 2.

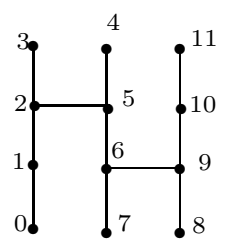

Figure 2: Strongly odd harmonious labeling of $T_{p^{-}}$tree with 12 vertices 
Theorem 2.2. If $T$ is a $T_{p}$-tree then the graph $T \hat{o} P_{m}$ is strongly odd harmonious.

Proof. Let $T$ be a $T_{p}$-tree with $n$ vertices. By definition there exists parallel transformation $P(T)$, we have $V(P(T))=V(T)$ and $E(P(T))=\left(E(T)-E_{d}\right) \cup E_{a}$, where $E_{d}$ is the set of deleted edges and $E_{a}$ is the newly added edges through the sequence $P=\left(P_{1}, P_{2}, \cdots, P_{l}\right)$ of the ept's used to obtain $P(T)$. Hence $E_{d}$ and $E_{a}$ have the same number of edges. Let $u_{1}, u_{2}, \cdots, u_{n}$ be the vertices of $P(T)$ successively, from one pendant vertex of $P(T)$ right up to the other. Let $x_{0}^{j}, x_{1}^{j}, \cdots, x_{m-1}^{j}$, $1 \leq j \leq n$ be the vertices of the $j^{t h}$ copy of $P_{m}$. Identify $x_{0}^{j}$ with $u_{j}$, where $1 \leq j \leq n$. Then the graph $T \hat{o} P_{m}$ has $n m$ vertices and $n m-1$ edges.

We define a labeling $f: V(G) \rightarrow\{0,1,2, \cdots, q=n m-1\}$ as follows:

$$
\begin{array}{lc}
f\left(u_{j}\right)=m j-1, & j=1,3, \cdots, n-1, \\
f\left(u_{j}\right)=m(j-1), & j=2,4, \cdots, n, \\
\text { For } 1 \leq i \leq m-1, f\left(x_{i}^{j}\right)=m j-i-1, & j=1,3, \cdots, n-1, \\
f\left(x_{i}^{j}\right)=m(j-1)+i, & j=2,4, \cdots, n .
\end{array}
$$

Let $\left(u_{i} u_{j}\right)$ be an edge of $T, 1 \leq i<j \leq n$. Let the ept $P_{1}$ delete the edge $\left(u_{i} u_{j}\right)$ and add the edge $\left(u_{i+t} u_{j-t}\right)$ where $t$ is the distance from $u_{i}$ to $u_{i+t}$ and also the distance from $u_{j}$ to $u_{j-t}$. Let the parallel transformation $P$ contain one of the constituent ept's $P_{1}$. Since $\left(u_{i+t} u_{j-t}\right)$ is an edge of $P(T)$, it follows that $i+t+1=j-t$, implies $j=i+2 t+1$. Therefore $i$ and $j$ are of opposite equivalence, that is, $i$ is even and $j$ is odd or vice-versa.

The induced edge label of $\left(u_{i} u_{j}\right)$ is given by

$$
\begin{aligned}
& f^{*}\left(u_{i} u_{j}\right)=f^{*}\left(u_{i} u_{i+2 t+1}\right)=f\left(u_{i}\right)+f\left(u_{i+2 t+1}\right)=2 m(i+t)-1, \\
& f^{*}\left(u_{i+t} u_{j-t}\right)=f^{*}\left(u_{i+t} u_{i+t+1}\right)=f\left(u_{i+t}\right)+f\left(u_{i+t+1}\right)=2 m(i+t)-1, \\
& f^{*}\left(u_{i} u_{j}\right)=f^{*}\left(u_{i+t} u_{j-t}\right) .
\end{aligned}
$$

The induced edge labels are

$$
\begin{array}{lc}
f^{*}\left(u_{j} u_{j+1}\right)=2 m j-1, & 1 \leq j \leq n-1, \\
\text { For } 1 \leq i \leq m-2, f^{*}\left(x_{i}^{j} x_{i+1}^{j}\right)=2 m j-2 i-3, & j=1,3, \cdots, n-1, \\
f^{*}\left(x_{i}^{j} x_{i+1}^{j}\right)=2 m(j-1)+2 i+1, & j=2,4, \cdots, n, \\
f^{*}\left(u_{j} x_{1}^{j}\right)=2 m j-3, & j=1,3, \cdots, n-1, \\
f^{*}\left(u_{j} x_{1}^{j}\right)=2 m(j-1)+i, & j=2,4, \cdots, n .
\end{array}
$$

Thus the induced edge labels are $1,3, \cdots, 2 m n-3$. Hence every $T \hat{o} P_{m}$ is strongly odd harmonious.

A strongly odd harmonious labeling of $T \hat{o} P_{4}$ where $T$ is a $T_{p}$-tree with 10 vertices is shown in Figure 3. 


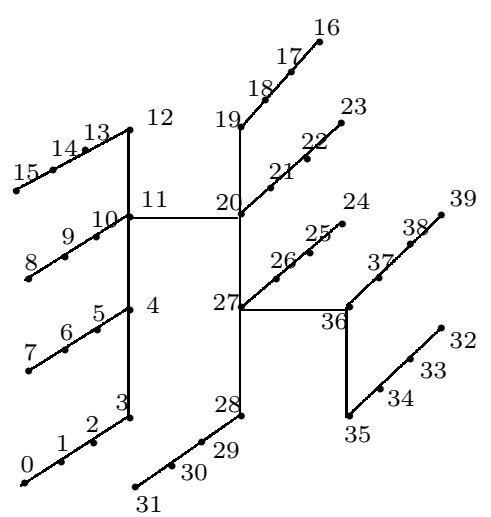

Figure 3: Strongly odd harmonious labeling of $T \hat{o} P_{4}$

Theorem 2.3. If $T$ is a $T_{p}$-tree then the graph $T \hat{o} 2 P_{m}$ is strongly odd harmonious.

Proof. Let $T$ be a $T_{p}$-tree with $n$ vertices. By definition there exists a parallel transformation $P(T)$, we have $V(P(T))=V(T)$ and $E(P(T))=\left(E(T)-E_{d}\right) \cup E_{a}$, where $E_{d}$ is the set of deleted edges and $E_{a}$ is the set of newly added edges through the sequence $P=\left(P_{1}, P_{2}, \cdots, P_{l}\right)$ of the ept's used to obtain $P(T)$. Hence $E_{d}$ and $E_{a}$ have the same number of edges. Let $x_{1,0}^{j}, x_{1,1}^{j}, x_{1,2}^{j}, \cdots, x_{1, m-1}^{j}$ and $x_{2,0}^{j}, x_{2,1}^{j}, x_{2,2}^{j}, \cdots, x_{2, m-1}^{j}, 1 \leq j \leq n$ be the vertices of two disjoint paths $P_{m}$. Identify $x_{1,0}^{j}$ and $x_{2,0}^{j}$ with $u_{j}, 1 \leq j \leq n$ to obtain $T \hat{o} 2 P_{m}$. Then the graph $T \hat{o} 2 P_{m}$ has $n(2 m-1)$ vertices and $n(2 m-1)-1$ edges.

We define a labeling $f: V(G) \rightarrow\{0,1,2, \cdots, q=n(2 m-1)-1\}$ as follows:
$f\left(u_{j}\right)=m-1+(2 m-1)(j-1)$,
if $j$ is odd,
$f\left(u_{j}\right)=3 m-2+(2 m-1)(j-2)$,
if $j$ is even,
$f\left(x_{1, i}^{j}\right)=m-1+(2 m-1)(j-1)-i$
if $j$ is odd,
$f\left(x_{1, i}^{j}\right)=3 m-2+(2 m-1)(j-2)-i$,
if $j$ is even,
$f\left(x_{2, i}^{j}\right)=m-1+(2 m-1)(j-1)+i$,
if $j$ is odd,
$f\left(x_{2, i}^{j}\right)=3 m-2+(2 m-1)(j-2)+i$,
if $j$ is even.

Let $\left(u_{i} u_{j}\right)$ be an edge of $T, 1 \leq i<j \leq n$. Let the ept $P_{1}$ delete the edge $\left(u_{i} u_{j}\right)$ and add the edge $\left(u_{i+t} u_{j-t}\right)$ where $t$ is the distance from $u_{i}$ to $u_{i+t}$ and also the distance from $u_{j}$ to $u_{j-t}$. Let the parallel transformation $P$ contain one of the constituent ept $s P_{1}$. Since $\left(u_{i+t} u_{j-t}\right)$ is an edge of $P(T)$, it follows that $i+t+1=j-t$, implies $j=i+2 t+1$. Therefore $i$ and $j$ are of opposite equivalence, that is, $i$ is even and $j$ is odd or vice-versa.

The induced edge label of $\left(u_{i} u_{j}\right)$ is given by

$f^{*}\left(u_{i} u_{j}\right)=f^{*}\left(u_{i} u_{i+2 t+1}\right)=f\left(u_{i}\right)+f\left(u_{i+2 t+1}\right)=4 m i+4 m t-2 i-2 t-1$,

$f^{*}\left(u_{i+t} u_{j-t}\right)=f^{*}\left(u_{i+t} u_{i+t+1}\right)=f\left(u_{i+t}\right)+f\left(u_{i+t+1}\right)=4 m i+4 m t-2 i-2 t-1$,

$f^{*}\left(u_{i} u_{j}\right)=f^{*}\left(u_{i+t} u_{j-t}\right)$. 
The induced edge labels are

$\begin{array}{ll}f^{*}\left(u_{j} x_{1,1}^{j}\right)=2(m-1)+2(2 m-1)(j-1)-1, & \text { if } j \text { is odd, } \\ f^{*}\left(u_{j} x_{1,1}^{j}\right)=2(3 m-2)+2(2 m-1)(j-2)-1, & \text { if } j \text { is even, } \\ f^{*}\left(u_{j} x_{2,1}^{j}\right)=2(m-1)+2(2 m-1)(j-1)+1, & \text { if } j \text { is odd, } \\ f^{*}\left(u_{j} x_{2,1}^{j}\right)=2(3 m-2)+2(2 m-1)(j-2)+1, & \text { if } j \text { is even, } \\ f^{*}\left(u_{j} u_{j+1}\right)=2(2 m-1)(j-1)+4 m+3, & 1 \leq j \leq n-1,\end{array}$

For $1 \leq i \leq m-2$,

$f^{*}\left(x_{1, i}^{j} x_{1, i+1}^{j}\right)=2(2 m-1)(j-1)+2(m-1)-2 i-1, \quad$ if $j$ is odd,

$f^{*}\left(x_{1, i}^{j} x_{1, i+1}^{j}\right)=2(2 m-1)(j-2)+2(3 m-2)-2 i-1, \quad$ if $j$ is even,

$f^{*}\left(x_{2, i}^{j} x_{2, i+1}^{j}\right)=2(2 m-1)(j-1)+2(m-1)+2 i+1, \quad$ if $j$ is odd,

$f^{*}\left(x_{2, i}^{j} x_{2, i+1}^{j}\right)=2(2 m-1)(j-2)+2(3 m-2)+2 i+1, \quad$ if $j$ is even.

Hence $T \hat{o} 2 P_{m}$ is strongly odd harmonious.

The strongly odd harmonious labeling of $T \hat{o} 2 P_{4}$ where $T$ is a $T_{p}$-tree with 13 vertices is shown in Figure 4.

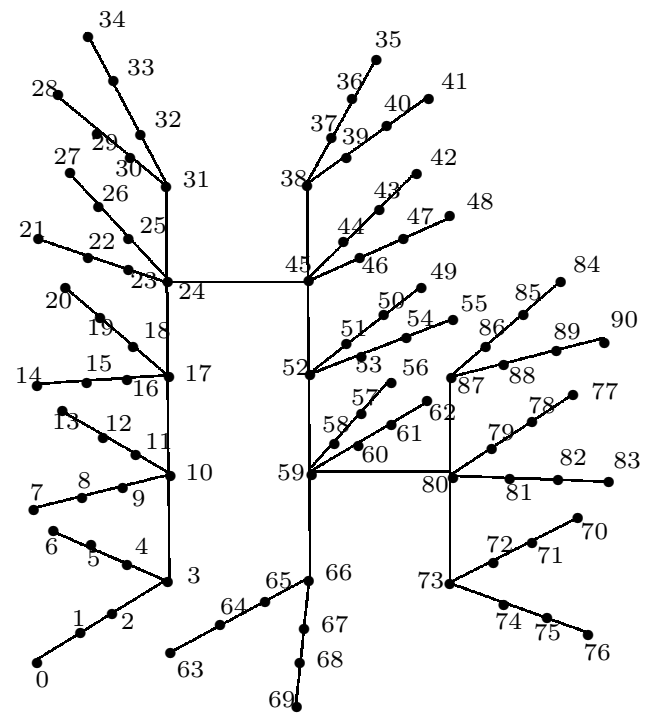

Figure 4: strongly odd harmonious labeling of $T \hat{o} 2 P_{4}$

Theorem 2.4. Every regular bamboo tree is odd harmonious.

Proof. Let $v_{0}^{j}, v_{1}^{j}, v_{2}^{j}, \cdots, v_{n-1}^{j}$ be the vertices of the $j^{\text {th }}$ path $P_{n}, 1 \leq j \leq m$ where $v_{0}^{j}$ is identified with the apex vertex $v_{0}$ and $v_{n-1}^{j}$ is identified with $u_{0}^{j}$ which is the centre of the $j^{\text {th }}$ star. Let $u_{1}^{j}, u_{2}^{j}, \cdots, u_{t}^{j}$ be the pendant vertices of the $j^{t h}$ star. The regular bamboo tree has $m(t+n-1)+1$ vertices and $m(t+n-1)$ edges. 
We define the labeling $f: V(G) \rightarrow\{0,1,2, \cdots, 2 m(n+t-1)-1\}$ as follows:

\section{Case(i): $m$ is odd}

$f\left(v_{0}\right)=0$,

For $1 \leq j \leq m$,

$f\left(v_{i}^{j}\right)=2 j-1+m(i-1)$

if $i$ is odd,

$f\left(v_{i}^{j}\right)=2+4(m-j)+m(i-2)$,

if $i$ is even,

$f\left(u_{i}^{j}\right)=m(n-1)+2 m-1+2 m(t-i)-2(m-j)$,

$1 \leq i \leq t$.

The induced edge labels are

For $1 \leq j \leq m$,

$f^{*}\left(v_{0} v_{1}^{j}\right)=2 j-1$

$f^{*}\left(v_{i}^{j} v_{i+1}^{j}\right)=2 j+2 m(i-1)+4(m-j)+1$,

if $i$ is odd,

$f^{*}\left(v_{i}^{j} v_{i+1}^{j}\right)=4(m-j)+2 m(i-1)+2 j+1$,

if $i$ is even,

$f^{*}\left(v_{n-1}^{j} u_{i}^{j}\right)=2 m(n+t-i)-2 j+1$,

$1 \leq i \leq t$.

Case (ii): $m$ is even

$f\left(v_{0}\right)=n-1$,

$f\left(v_{i}^{1}\right)=n-1-i$,

$1 \leq i \leq n-1$

For $2 \leq j \leq m$ and $1 \leq i \leq t$,

$f\left(v_{i}^{j}\right)=n+2(j-2)+(m-1)(i-1), \quad$ if $i$ is odd,

$f\left(v_{i}^{j}\right)=n+1+4(m-j)+(m-1)(i-2), \quad$ if $i$ is even,

If $n$ is odd, $f\left(u_{i}^{1}\right)=2 m n+2(m-1)(t-1)-2 m+2(t-i)+7$,

If $n$ is even, $f\left(u_{i}^{1}\right)=2 m n+2(m-1)(t-1)+2(t-i)-1$,

If $n$ is odd, $f\left(u_{i}^{j}\right)=m(n-1)+5+2(m-1)(t-i)-2(m-j)$,

If $n$ is even, $f\left(u_{i}^{j}\right)=m(n-2)+3+2(m-1)(t-i)+4(m-j)$.

The induced edge labels are

$f^{*}\left(v_{0} v_{1}^{1}\right)=2 n-3$,

$f^{*}\left(v_{0} v_{1}^{j}\right)=2 n-1+2(j-2)$,

$2 \leq j \leq m$,

$f^{*}\left(v_{i}^{j} v_{i+1}^{j}\right)=2 n+2(j-2)+2(m-1)(i-1)+4(m-j)+1$,

$2 \leq j \leq m$.

For $2 \leq j \leq m$ and $1 \leq i \leq t$,

If $n$ is even, $f^{*}\left(v_{n-1}^{j} u_{i}^{j}\right)=2 m n-2 j+1+2(m-1)(t-i)$.

If $n$ is odd, $f^{*}\left(v_{n-1}^{j} u_{i}^{j}\right)=2 m(n-1)-2 j+2(m-1)(t-i)+9$.

$f^{*}\left(v_{n-1}^{1} u_{i}^{1}\right)= \begin{cases}2 m n+2(m-1)(t-1)-2 m+2(t-i)+7 & \text { if } n \text { is odd } \\ 2 m n+2(m-1)(t-1)+2(t-i)-1 & \text { if } n \text { is even. }\end{cases}$

Thus every regular bamboo tree is odd harmonious.

An odd harmonious labeling of a regular bamboo tree with $m=5, n=6, t=2$ is shown in Figure 5 . 


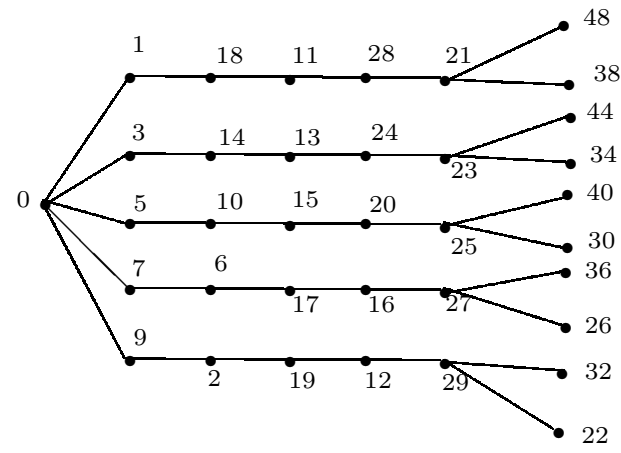

Figure 5: A regular bamboo tree with $m=5, n=6, t=2$

An odd harmonious labeling of a regular bamboo tree with $m=4, n=5, t=2$ is shown in Figure 6 .

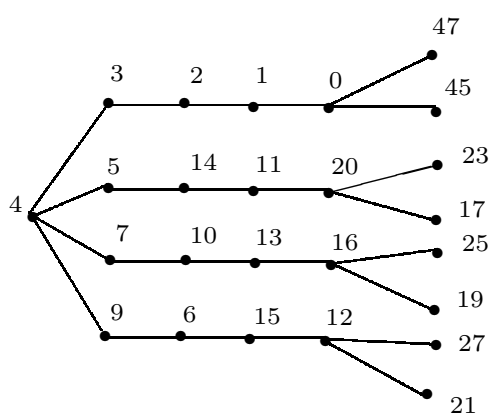

Figure 6: A regular bamboo tree with $m=4, n=5, t=2$

Theorem 2.5. The graph $C_{n} \hat{o} P_{m}, n \equiv 0(\bmod 4)$ is odd harmonious.

Proof. Let $u_{1}, u_{2}, \cdots, u_{n}$ be the vertices of cycle $C_{n}$. Let $u_{i}^{0}, u_{i}^{1}, \cdots, u_{i}^{m-1}$ be the vertices of path $P_{m}$. We identify $u_{i}^{0}$ with $u_{i}, 1 \leq i \leq n$ to obtain $C_{n} \hat{o} P_{m}$. Then the graph $C_{n} \hat{o} P_{m}$ has $m n$ edges and vertices.

We define the labeling $f: V(G) \rightarrow\{0,1,2, \cdots, 2 n m-1\}$ as follows:

Case (i): $m$ is odd

$$
\begin{aligned}
& f\left(u_{i}\right)=m i, \\
& f\left(u_{i}\right)=m i-m-1, \\
& f\left(u_{i}\right)=m i-m+1, \\
& f\left(u_{i}^{j}\right)=m i-j,
\end{aligned}
$$

$$
\begin{aligned}
& i=1,3, \cdots, n-1, \\
& i=2,4, \cdots, \frac{n}{2}, \\
& i=\frac{n}{2}+2, \cdots, n \\
& \text { if } i \text { is odd and } j \text { is even, }
\end{aligned}
$$


$f\left(u_{i}^{j}\right)=\left\{\begin{array}{ll}m i-j-2 & \text { if } 1 \leq i \leq \frac{n}{2}-1 \\ m i-j & \text { if } \frac{n}{2}+1 \leq i \leq n-1\end{array} \quad\right.$ if both $i$ and $j$ are odd,

$f\left(u_{i}^{j}\right)=\left\{\begin{array}{ll}m i-m+j-1 & \text { if } 2 \leq i \leq \frac{n}{2} \\ m i-m+j+1 & \text { if } \frac{n}{2}+2 \leq i \leq n\end{array} \quad\right.$ if both $i$ and $j$ are even,

$f\left(u_{i}^{j}\right)=m i-m+j+1$,

if $i$ is even and $j$ is odd.

The induced edge labels are

$f^{*}\left(u_{i} u_{i+1}\right)=2 i m-1$,

$f^{*}\left(u_{i} u_{i+1}\right)=2 i m+1$,

$f^{*}\left(u_{1} u_{n}\right)=m n+1$,

$f\left(u_{i} u_{i}^{1}\right)= \begin{cases}2 m i-3 & \text { if } 1 \leq i \leq \frac{n}{2}-1 \\ 2 m i-1 & \text { if } \frac{n}{2}+1 \leq i \leq n-1\end{cases}$

$f\left(u_{i} u_{i}^{1}\right)=\left\{\begin{array}{ll}2 m i-2 m+1 & \text { if } 2 \leq i \leq \frac{n}{2} \\ 2 m i-2 m+3 & \text { if } \frac{n}{2}+2 \leq i \leq n\end{array} \quad\right.$ if $i$ is even and $j$ is odd,

$f\left(u_{i}^{j} u_{i}^{j+1}\right)=\left\{\begin{array}{ll}2 m i-2 j-3 & \text { if } 1 \leq i \leq \frac{n}{2}-1 \\ 2 m i-2 j-1 & \text { if } \frac{n}{2}+1 \leq i \leq n-1\end{array} \quad\right.$ if $i$ is odd

$f\left(u_{i}^{j} u_{i}^{j+1}\right)=\left\{\begin{array}{ll}2 m i-2 m+2 j-1 & \text { if } 2 \leq i \leq \frac{n}{2} \\ 2 m i-2 m+2 j+1 & \text { if } \frac{n}{2}+2 \leq i \leq n\end{array} \quad\right.$ if $i$ is even.

Case (ii): $m$ is even

$f\left(u_{i}\right)=m i-2$,

$f\left(u_{i}\right)= \begin{cases}m i-m+1 & \text { if } 2 \leq i \leq \frac{n}{2} \\ m i-m+3 & \text { if } \frac{n}{2}+2 \leq i \leq n\end{cases}$

$i=1,3, \cdots, n-1$,

$f\left(u_{i}^{j}\right)= \begin{cases}m i-j & \text { if } 1 \leq i \leq \frac{n}{2}-1 \\ m i-j+2 & \text { if } \frac{n}{2}+1 \leq i \leq n-1\end{cases}$

if $i$ is even,

$f\left(u_{i}^{j}\right)=m i-j-2, \quad 1 \leq i \leq n-1$,

$$
\begin{aligned}
& 1 \leq i \leq \frac{n}{2} \\
& \frac{n}{2}+1 \leq i \leq n-1,
\end{aligned}
$$

$f\left(u_{i}^{j}\right)=m i-m+j-1,2 \leq i \leq n-2$,

$f\left(u_{i}^{j}\right)= \begin{cases}m i-m+j+1 & \text { if } 2 \leq i \leq \frac{n}{2} \\ m i-m+j-1 & \text { if } \frac{n}{2}+2 \leq i \leq n\end{cases}$

The induced edge labels are

$f^{*}\left(u_{i} u_{i+1}\right)= \begin{cases}2 m i-1 & \text { if } 1 \leq i \leq \frac{n}{2} \\ 2 m i+1 & \text { if } \frac{n}{2}+1 \leq i \leq n\end{cases}$

if $i$ is even and $j$ is odd,

$f^{*}\left(u_{1} u_{n}\right)=m n+1$

$f\left(u_{i} u_{i}^{1}\right)= \begin{cases}2 m i-3 & \text { if } 1 \leq i \leq \frac{n}{2}-1 \\ 2 m i-1 & \text { if } \frac{n}{2}+1 \leq i \leq n-1\end{cases}$

if both $i$ and $j$ are odd,

if $i$ is odd and $j$ is even,

if $i$ is even and $j$ is odd,

if both $i$ and $j$ are even.

if $i$ is odd, 
$f^{*}\left(u_{i} u_{i}^{1}\right)= \begin{cases}2 m i-2 m+1 & \text { if } 2 \leq i \leq \frac{n}{2} \\ 2 m i-2 m+3 & \text { if } \frac{n}{2}+2 \leq i \leq n\end{cases}$

if $i$ is even,

$f^{*}\left(u_{i}^{j} u_{i}^{j+1}\right)=\left\{\begin{array}{ll}2 m i-2 j-3 & \text { if } 1 \leq i \leq \frac{n}{2}-1 \\ 2 m i-2 j-1 & \text { if } \frac{n}{2}+1 \leq i \leq n-1\end{array} \quad\right.$ if $i$ is odd,

$f^{*}\left(u_{i}^{j} u_{i}^{j+1}\right)=\left\{\begin{array}{ll}2 m i-2 m+2 j+1 & \text { if } 2 \leq i \leq \frac{n}{2} \\ 2 m i-2 m+2 j-1 & \text { if } \frac{n}{2}+2 \leq i \leq n\end{array} \quad\right.$ if $i$ is even.

Therefore $C_{n} \hat{o} P_{m}$ is odd harmonious.

An odd harmonious labeling of $C_{4} \hat{o} P_{5}$ and $C_{12} \hat{o} P_{4}$ are shown in Figure 7 .
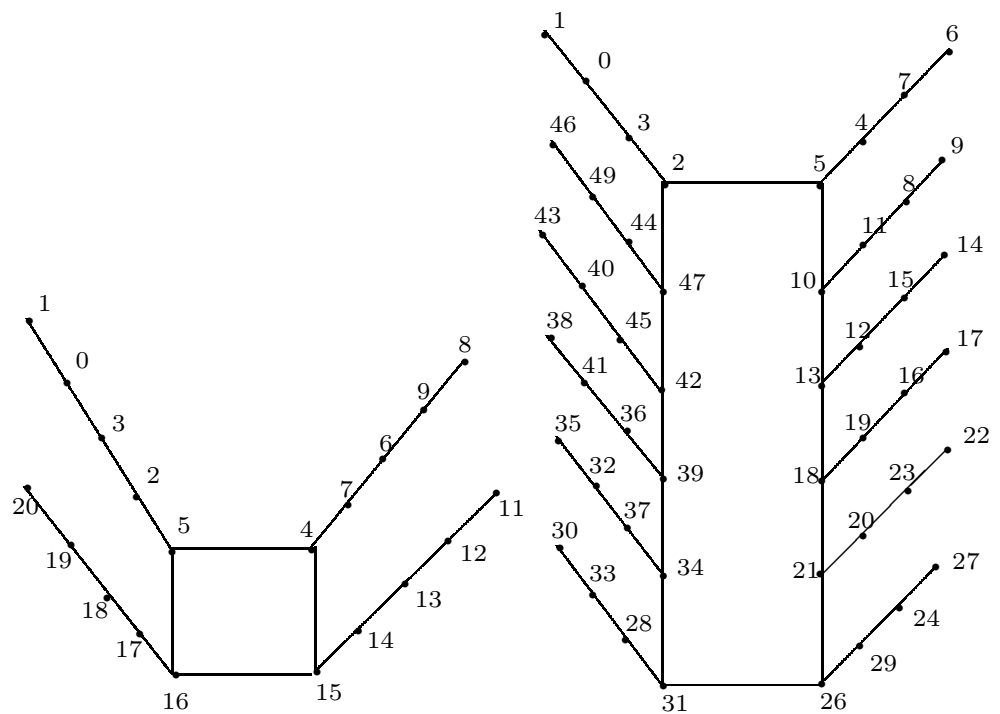

Figure 7: An odd harmonious labeling of $C_{4} \hat{o} P_{5}$ and $C_{12} \hat{o} P_{4}$

Theorem 2.6. The graph $C_{n} \hat{o} 2 P_{m}, n \equiv 0(\bmod 4)$ is odd harmonious.

Proof. Let $u_{1}, u_{2}, \cdots, u_{n}$ be the vertices of $C_{n}$. Let $x_{1,0}^{j}, x_{1,1}^{j}, x_{1,2}^{j}, \cdots, x_{1, m-1}^{j}$ and $x_{2,0}^{j}, x_{2,1}^{j}$, $x_{2,2}^{j}, \cdots, x_{2, m-1}^{j}, 1 \leq j \leq n$ be the vertices of two disjoint paths $P_{m}$. Identify $x_{1,0}^{j}$ and $x_{2,0}^{j}$ with $u_{j}, 1 \leq j \leq n$ to obtain $C_{n} \hat{\circ} 2 P_{m}$. Then the graph $C_{n} \hat{\circ} 2 P_{m}$ has $n(2 m-1)$ edges and vertices.

We define the labeling $f: V(G) \rightarrow\{0,1,2, \cdots, 2 n(2 m-1)-1\}$ as follows:

$f\left(u_{j}\right)=m-1+(2 m-1)(j-1)$,

$f\left(u_{j}\right)= \begin{cases}3 m-2+(2 m-1)(j-2) & \text { if } 2 \leq j \leq \frac{n}{2} \\ 7 m-2+(2 m-1)(j-4) & \text { if } \frac{n}{2}+2 \leq j \leq n\end{cases}$

$j=1,3, \cdots, n-1$,

$f\left(x_{1, i}^{j}\right)=(m-1)+(2 m-1)(j-1)-i$,

if $j$ is even,

if $i$ is even and $j$ is odd, 
$f\left(x_{1, i}^{j}\right)=\left\{\begin{array}{ll}m+(2 m-1)(j-1)-i-1 & \text { if } 1 \leq j \leq \frac{n}{2}-1 \\ m+(2 m-1)(j-1)-i+1 & \text { if } \frac{n}{2}+1 \leq j \leq n-1\end{array} \quad\right.$ if both $i$ and $j$ are odd,

$f\left(x_{2, i}^{j}\right)=m+(2 m-1)(j-1)+i-1,1 \leq j \leq n-1$, if $i$ is even and $j$ is odd, $f\left(x_{2, i}^{j}\right)=\left\{\begin{array}{ll}m+(2 m-1)(j-1)+i-1 & \text { if } 1 \leq j \leq \frac{n}{2}-1 \\ m+(2 m-1)(j-1)+i+1 & \text { if } \frac{n}{2}+1 \leq j \leq n-1\end{array} \quad\right.$ if both $i$ and $j$ are odd,

$f\left(x_{1, i}^{j}\right)=\left\{\begin{array}{ll}3 m+(2 m-1)(j-2)-i-2 & \text { if } 2 \leq j \leq \frac{n}{2} \\ 7 m+(2 m-1)(j-4)-i-2 & \text { if } \frac{n}{2}+2 \leq j \leq n\end{array}\right.$ if both $i$ and $j$ are even, $f\left(x_{1, i}^{j}\right)=\left\{\begin{array}{ll}3 m+(2 m-1)(j-2)-i-2 & \text { if } 2 \leq j \leq \frac{n}{2} \\ 7 m+(2 m-1)(j-4)-i-4 & \text { if } \frac{n}{2}+2 \leq j \leq n\end{array}\right.$ if $i$ is odd and $j$ is even, $f\left(x_{2, i}^{j}\right)=\left\{\begin{array}{ll}3 m+(2 m-1)(j-2)+i-2 & \text { if } 2 \leq j \leq \frac{n}{2} \\ 7 m+(2 m-1)(j-4)+i-2 & \text { if } \frac{n}{2}+2 \leq j \leq n\end{array}\right.$ if both $i$ and $j$ are even, $f\left(x_{2, i}^{j}\right)=\left\{\begin{array}{ll}3 m+(2 m-1)(j-2)+i-2 & \text { if } 2 \leq j \leq \frac{n}{2} \\ 7 m+(2 m-1)(j-4)+i-4 & \text { if } \frac{n}{2}+2 \leq j \leq n\end{array}\right.$ if $i$ is odd and $j$ is even.

The induced edge labels are

$$
\begin{aligned}
& f^{*}\left(u_{j} u_{j+1}\right)=4 m+2(2 m-1)(j-1)-3, \\
& 1 \leq j \leq \frac{n}{2}-1 \\
& f^{*}\left(u_{j} u_{j+1}\right)=8 m+2(2 m-1)(j-2)-3, \\
& f^{*}\left(u_{j} x_{1,1}^{j}\right)=\left\{\begin{array}{ll}
2 m+2(2 m-1)(j-1)-3 & \text { if } 1 \leq j \leq \frac{n}{2}-1 \\
2 m+2(2 m-1)(j-1)-1 & \text { if } \frac{n}{2}+1 \leq j \leq n-1
\end{array} \text { if } j\right. \text { is odd, } \\
& f^{*}\left(u_{j} x_{1,1}^{j}\right)=\left\{\begin{array}{ll}
6 m+2(2 m-1)(j-2)-5 & \text { if } 2 \leq j \leq \frac{n}{2} \\
14 m+2(2 m-1)(j-4)-7 & \text { if } \frac{n}{2}+2 \leq j \leq n
\end{array} \quad \text { if } j\right. \text { is even, } \\
& f^{*}\left(u_{j} x_{2,1}^{j}\right)=\left\{\begin{array}{ll}
2 m+2(2 m-1)(j-1)-1 & \text { if } 1 \leq j \leq \frac{n}{2}-1 \\
2 m+2(2 m-1)(j-1)+1 & \text { if } \frac{n}{2}+1 \leq j \leq n-1
\end{array} \quad \text { if } j\right. \text { is odd } \\
& f^{*}\left(u_{j} x_{2,1}^{j}\right)=\left\{\begin{array}{ll}
6 m+2(2 m-1)(j-2)-3 & \text { if } 2 \leq j \leq \frac{n}{2} \\
14 m+2(2 m-1)(j-4)-5 & \text { if } \frac{n}{2}+2 \leq j \leq n
\end{array} \quad \text { if } j\right. \text { is even, } \\
& f^{*}\left(x_{1, i}^{j} x_{1, i+1}^{j}\right)=\left\{\begin{array}{ll}
4 m j-2 m-2 j-2 i-1 & \text { if } 1 \leq j \leq \frac{n}{2}-1 \\
4 m j-2 m-2 j-2 i+1 & \text { if } \frac{n}{2}+1 \leq j \leq n-1
\end{array} \quad \text { if } j\right. \text { is odd, } \\
& f^{*}\left(x_{1, i}^{j} x_{1, i+1}^{j}\right)=\left\{\begin{array}{ll}
6 m+2(2 m-1)(j-2)-2 i-5 & \text { if } 2 \leq j \leq \frac{n}{2} \\
14 m+2(2 m-1)(j-4)-2 i-7 & \text { if } \frac{n}{2}+2 \leq j \leq n
\end{array} \text { if } j\right. \text { is even, } \\
& f^{*}\left(x_{2, i}^{j} x_{2, i+1}^{j}\right)=\left\{\begin{array}{ll}
2 m+2(2 m-1)(j-1)+2 i-1 & \text { if } 1 \leq j \leq \frac{n}{2}-1 \\
2 m+2(2 m-1)(j-1)+2 i+1 & \text { if } \frac{n}{2}+1 \leq j \leq n-1
\end{array} \text { if } j\right. \text { is odd, }
\end{aligned}
$$


$f^{*}\left(x_{2, i}^{j} x_{2, i+1}^{j}\right)=\left\{\begin{array}{ll}6 m+2(2 m-1)(j-2)+2 i-3 & \text { if } 2 \leq j \leq \frac{n}{2} \\ 14 m+2(2 m-1)(j-4)+2 i-5 & \text { if } \frac{n}{2}+2 \leq j \leq n\end{array} \quad\right.$ if $j$ is even, $f^{*}\left(u_{1} u_{n}\right)=8 m+(2 m-1)(n-4)-3$.

Thus $C_{n} \hat{\circ} 2 P_{m}$ is odd harmonious.

An odd harmonious labeling of $C_{8} \hat{o} 2 P_{3}$ is shown in Figure 8 .



Figure 8: An odd harmonious labeling of $C_{8} \hat{o} 2 P_{3}$

Theorem 2.7. Every subdivided grid $P_{m} \times P_{m}, m \geq 2$ is strongly odd harmonious.

Proof. Let $v_{i, 1}, v_{i, 2}, \cdots, v_{i, m}, 1 \leq i \leq m$ be the vertices of the $i^{\text {th }}$ row of $P_{m} \times P_{m}$. Let $u_{1, i}$, $u_{2, i}, \cdots, u_{m-1, i}, 1 \leq i \leq m$ be the vertices of the subdivided of $i^{t h}$ column and $w_{1, i}, w_{2, i}, \cdots, w_{m-1, i}$, $1 \leq i \leq m$ be the vertices of the subdivided of $i^{t h}$ row. Then the subdivided grid graph has $m(3 m-2)$ and $4 m(m-1)$ vertices and edges respectively.

We define a labeling $f: V(G) \rightarrow\{0,1,2, \cdots, q=4 m(m-1)\}$ as follows:

$$
\begin{array}{lrl}
f\left(v_{i, j}\right)=2(j-1)+2(2 m-1)(i-1), & 1 \leq j \leq m \text { and } i \text { is odd, } \\
f\left(v_{i, j}\right)=2(3 m-j-1)+2(2 m-1)(i-2), & 1 \leq j \leq m \text { and } i \text { is even, } \\
f\left(u_{i, j}\right)=2 m-1+4(m-j)+2(2 m-1)(i-1), & 1 \leq j \leq m \text { and } i \text { is odd, } \\
f\left(u_{i, j}\right)=6 m-3+4(j-1)+2(2 m-1)(i-2), & 1 \leq j \leq m \text { and } i \text { is even, } \\
f\left(w_{i, 1}\right)=2 i-1, & 1 \leq i \leq m-1, \\
f\left(w_{i, j}\right)=(m-1)(4 j-2)-4 i+2 j-1, & 1 \leq i \leq m-1 \text { and } j \text { is even, } \\
f\left(w_{i, j}\right)=(m-1)(4 j-6)+4 i+2 j-5, & 1 \leq i \leq m-1 \text { and } j \text { is odd. }
\end{array}
$$


The induced edge labels are

$f^{*}\left(v_{i, j} u_{i, j}\right)=2(j-1)+4(2 m-1)(i-1)+2 m-1+4(m-j), \quad 1 \leq j \leq m$ and $i$ is odd, $f^{*}\left(u_{i, j} v_{i+1, j}\right)=2 m-1+2(5 m-3 j-1)+4(2 m-1)(i-1), \quad 1 \leq j \leq m$ and $i$ is odd, $f^{*}\left(u_{i, j} v_{i+1, j}\right)=6 m-3+6(j-1)+4(2 m-1)(i-1), \quad 1 \leq j \leq m$ and $i$ is even, $f^{*}\left(v_{i, j} w_{t, k}\right)=2(j-1)+2(2 m-1)(i-1)+(m-1)(4 k-2)-4 t-2 k-1, i$ is odd and $k$ is even, $f^{*}\left(v_{i, j} w_{t, k}\right)=2(j-1)+2(2 m-1)(i-1)+(m-1)(4 k-6)+4 t+2 k-5, i$ is odd and $k$ is odd, $f^{*}\left(v_{i, j} w_{t, k}\right)=2(3 m-j-1)+2(2 m-1)(i-2)+2(m-1)(2 k-1)-4 t-2 k-1, i$ is even and $k$ is even,

$f^{*}\left(v_{i, j} w_{t, k}\right)=2(3 m-j-1)+2(2 m-1)(i-2)+2(m-1)(2 k-3)+4 t+2 k-5, i$ is even and $k$ is odd,

$$
\begin{aligned}
& f^{*}\left(v_{1, j} w_{i, 1}\right)=2(j-1)+2 i-1, \quad 1 \leq i \text { and } j \leq m-1, \\
& f^{*}\left(w_{i, 1} v_{1, j}\right)=2(j-1)+2 i-1, \quad 1 \leq i \leq m-1 \text { and } 2 \leq j \leq m \text {. }
\end{aligned}
$$

Therefore every subdivided grid graph is strongly odd harmonious.

A strongly odd harmonious labeling of subdivided grid $P_{4} \times P_{4}$ is shown in Figure 9 .

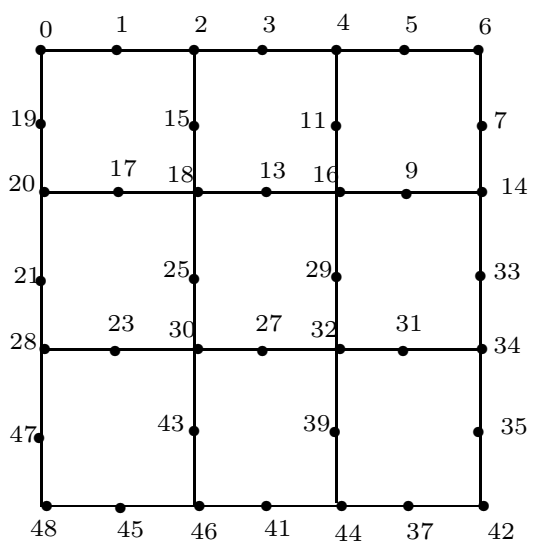

Figure 9: Strongly odd harmonious labeling of subdivided grid $P_{4} \times P_{4}$

\section{Acknowledgements}

The authors thank the referees for their useful comments to improve the presentation of the paper. 


\section{References}

[1] J. A. Gallian, "A dynamic survey of graph labeling", The Electronic Journal of Combinatorics, \# DS6, 2019.

[2] R. L. Graham and N. J. A. Sloane, "On additive bases and harmonious graphs", SIAM J. Algebraic Discrete Methods, vol. 1, no. 4, pp. 382-404, 1980.

[3] F. Harary, Graph theory, Addison-Wesley, Massachusetts, 1972.

[4] S. M. Hegde and S. Shetty, "On graceful trees", Appl. Math. E-Notes, vol. 2, pp. 192-197, 2002.

[5] P. Jeyanthi, S.Philo and Kiki A. Sugeng, "Odd harmonious labeling of some new families of graphs", SUT Journal of Mathematics, vol. 51, no. 2, pp. 181-193, 2015.

[6] P. Jeyanthi and S. Philo, "Odd harmonious labeling of some cycle related graphs", Proyecciones Journal of Mathematics, vol. 35, no. 1, pp. 85-98, 2016.

[7] P. Jeyanthi and S. Philo, "Odd harmonious labeling of plus graphs", Bull. Int. Math. Virtual Inst., vol. 7, no. 3, pp. 515-526, 2017.

[8] P. Jeyanthi, S. Philo and M. K. Siddiqui, "Odd harmonious labeling of super subdivision graphs", Proyecciones Journal of Mathematics, vol. 38, no. 1, pp. 1-11, 2019.

[9] P. Jeyanthi and S. Philo, "Odd harmonious labeling of subdivided shell graphs", International Journal of Computer Sciences and Engineering, vol. 7, no. 5, pp. 77-80, 2019.

[10] P. Jeyanthi and S. Philo, "Odd harmonious labeling of certain graphs", Journal of Applied Science and Computations, vol. 6 no. 4, pp. 1224-1232, 2019.

[11] P. Jeyanthi and S. Philo, "Some results on odd harmonious labeling of graphs", Bull. Int. Math. Virtual Inst., vol. 9, no. 3, pp. 567-576, 2019.

[12] P. Jeyanthi and S. Philo, "Odd harmonious labeling of some new graphs", Southeast Asian Bull. Math., vol. 43, no. 4, pp. 509-523, 2019.

[13] P. Jeyanthi, S. Philo and M. Youssef, "Odd harmonious labeling of grid graph", Proyecciones Journal of Mathematics, vol. 38, no. 3, pp. 411-429, 2019.

[14] Z. H. Liang and Z. L. Bai, "On the odd harmonious graphs with applications", J. Appl. Math. Comput., vol. 29, no. 1-2, pp. 105-116, 2009.

[15] G. Pooranam, R. Vasuki and S. Suganthi, "Even vertex odd mean labeling of transformed trees", SUT J. Math., vol. 52, no. 2, pp. 117-131, 2016. 
[16] V. Ramalakshmi, C. Sekar, "One modulo $N$ gracefulness of regular bamboo tree and coconut tree", International Journal on Applications of Graph Theory in wireless Ad Hoc Networks and Sensor Networks, vol. 6, no. 2, pp. 1-10, 2014.

[17] P. Selvaraju, P. Balaganesan and J.Renuka, "Odd harmonious labeling of some path related graphs", International J. of Math. Sci. and Engg. Appls., vol. 7, no. III, pp. 163-170, 2013.

[18] S. K. Vaidya and N. H. Shah, "Some new odd harmonious graphs", International Journal of Mathematics and Soft Computing, vol. 1, pp. 9-16, 2011.

[19] S. K. Vaidya, N. H. Shah, "Odd harmonious labeling of some graphs", International J.Math. Combin., vol. 3, pp. 105-112, 2012. 